\title{
New Materials of 3D Printing
}

\author{
Hao Wu ${ }^{1, a . *}$ and Cuiqiao $\mathrm{Li}^{2, \mathrm{~b}}$
}

${ }^{1}$ Key Laboratory of Metallurgical Equipment and Control Technology, Ministry of Education, Wuhan University of Science and Technology, Wuhan 430081, China;

${ }^{2}$ Hubei Key Laboratory of Mechanical Transmission and Manufacturing Engineering, Wuhan University of Science and Technology, Wuhan 430081, China;

a1252334436@qq.com, b1137410667@qq.com

* the corresponding author

Keyword: 3D printing; New Materials; Metallic materials; Hybrid printing.

\begin{abstract}
D printing is one of the rapid prototyping technologies and is considered as one of the core technologies of the third industrial revolution. However, the current development of 3D printing materials has limited the development of 3D printing technology. In the field of 3D printing technology, due to its low melting point and low cost, polymer materials are the fastest growing and the most widely used in 3D printing. However, due to its high melting point, metallic materials have been slow to develop in 3D printing technology. In this paper, 3D printing is firstly introduced by introducing a low-melting-point metal alloy material to explain the difficulties of 3D metal printing. Then, by introducing a method that is compatible with mixed 3D printing of metallic materials and nonmetallic materials, and through research, it is proved that new metallic materials and nonmetal materials have good compatibility. Finally, this hybrid 3D printing method provides a new direction for the future development of 3D printing technology.
\end{abstract}

\section{Introduction}

3D printing technology is a kind of rapid prototyping technology. As a rapidly developing technology, it has the prospect of universal application in chemical synthesis, home appliances and electronic circuit devices. The basic method of rapid prototyping is to create a three-dimensional entity by laying up continuous layers of material of powdered plastic, metal particles, or other binder materials. Commonly used in 3D printing technology printing materials include engineering plastics, photosensitive resins, rubber materials, metal materials, the shape of the powder, lamellar, liquid, etc. 0 . The current constraints of 3D printing technology development are two factors that are print processes and materials used for printing, the most rapid development of the material in the $3 \mathrm{D}$ printing technology belongs to plastic as the representative of the polymer material, they have a lower melting point, preferably thermoplastic, excellent liquidity and rapid cooling of the adhesive characteristics. In the field of metal printing, 3D printing has been slow due to the fact that metal printing usually takes a long time and lacks high-performance printable materials 0 . In the field of hybrid printing of metallic materials and non-metallic materials, currently available 3D printing technologies can't simultaneously print metallic and nonmetallic materials due to their large melting point differences and poor compatibility with each other. Due to material constraints, 3D printing has developed into a bottleneck and we should work harder on new materials to solve the current obstacles. This article discusses common polymer materials and new alloys that can be used for co-printing with non-metallic materials.

\section{Polymer Materials}

Engineering Plastics. Engineering plastics is the most widely used 3D printing materials, these materials have high strength, good heat resistance and impact resistance, etc., so print out this type of raw material products widely used in industrial and civil applications, usually there are engineering plastics like ABS, PC, nylons, etc. 0. ABS material is the preferred material for (Fused 
Deposition Modeling) 3D printing processes and nearly 90\% of FDM models are made from this material. The heat-resistant temperature of $93.3^{\circ} \mathrm{C}$, with high strength, toughness and impact resistance is also better, and the color and type of ABS materials are many in the mechanical processing, electroplating and automotive, consumer electronics products, such as the general application . PC material is a kind of thermoplastic material, its heat-resistant temperature is $125^{\circ} \mathrm{C}$, all the characteristics of PC material possesses are reflected in the engineering plastic. PC materials, a single color, but the intensity higher than the ABS material about $60 \%$, are widely used in electronic circuits, consumer electronics, automotive interiors, medical and medical equipment and other fields. Nylon-like material is a kind of white powder, the heat-resisting temperature is $110^{\circ} \mathrm{C}$, relative to the general plastic, the heat distortion temperature, the bending strength and so on all has the enhancement, but the material shrinkage rate reduces, has the good cohesiveness, and It is easier to preform into spherical fine particles with uniform particle size. However, the surface of nylon material is roughened and its impact hardness is reduced. It is applied to automobiles, electric circuits and so on 0 .

Photosensitive Resin. Photosensitive resin that Ultraviolet Rays (UV) resin, is a stable liquid printing raw materials, resins are generally composed of monomer polymers, prepolymers and UV initiated extrusion, during printing, irradiated with ultraviolet light through a particular wavelength (250-300mm) curing can be completed immediately. Therefore, such materials have better printing performance is dry, smooth surface after forming a smooth, high resolution, product quality even more than injection products. These advantages make the photosensitive resin material is the preferred raw art 3D printed article, but the high cost of the photosensitive resin, and the mechanical strength, heat resistance engineering plastics and so below, thus affecting the application of the material in a considerable range.

Rubber Materials. Rubber material is a material with a variety of levels of elasticity, the material has a tear strength, hardness, tensile strength, etc., so that the rubber-based raw materials printed products are very suitable for non-slip or soft surface requirements, 3D printed rubber products are mainly used in consumer electronics products, automotive interiors and tires.

\section{Metal Printing Material Research}

Metal 3D Printing Method. So far, laser sintering (LS), laser melting (LM) and laser metal deposition (LMD) are three typical 3D metal printing methods that are commonly used to process a wide range of refractory metals, alloys and various metal composites. In order to achieve a favorable metallic structure in these manufacturing, it is necessary to select a suitable powder material and a laser process. Due to these limitations, the types of printable metallic materials currently available at room temperature are quite limited if using conventional 3D metal printing methods. Pneumatic liquid metal 3D printing has many advantages over laser printing methods in terms of low cost, ease of deposition on different substrates, and the direct implementation of hybrid printing for the final product 0 .

Obviously, the choice of a suitable melting point candidate metal material is to determine the aerodynamic liquid metal 3D printing is the key to the smooth operation. In recent years, low-melting-point metal materials are gaining more and more attention in the field of electronics and 3D printing. In some researches, 3D metal printing mainly uses GaIn2.4 alloy and eutectic alloy made of gallium and indium as metal printing material, the advantage of using these alloys is that their melting point $\left(15.7^{\circ} \mathrm{C}\right)$ is very low for printing. The only pity is that the melting point of this alloy is too low. The use of this material in metal printing can result in melting of the 3D metal object at room temperature. In this regard, the melting point of the metal material should be greater than room temperature, but for practical operation, its melting point can't be too high.

Metal 3D Printing Technology. In order to further extend 3D printing technology for manufacturing metal objects, an alternative method of liquid phase 3D printing is proposed. To ensure print quality 3D printing liquid, system liquid droplet formation studied, and several other basic concepts of the transition nozzle fluid mechanics problems. When liquid metal is injected through a needle into another immiscible liquid, two types of droplet formation mechanism will be 
observed 0 . If the speed of liquid metal injection is less than a certain critical value, the droplets will form directly at the tip; if the injection rate is greater than the critical value, the liquid metal will form an injection stream and then break down into droplets due to instability. The exterior photo of the printing head is presented in the inset of Fig. 1. In general, in the former case, the droplet size is determined jointly by buoyancy, viscosity, surface tension and fluid inertia, while in the latter case the droplet size is determined by the jetting stability kinetics. In addition, the basic characteristics of droplet detachment from the tip will also affect the printing process and the final print quality 0 .

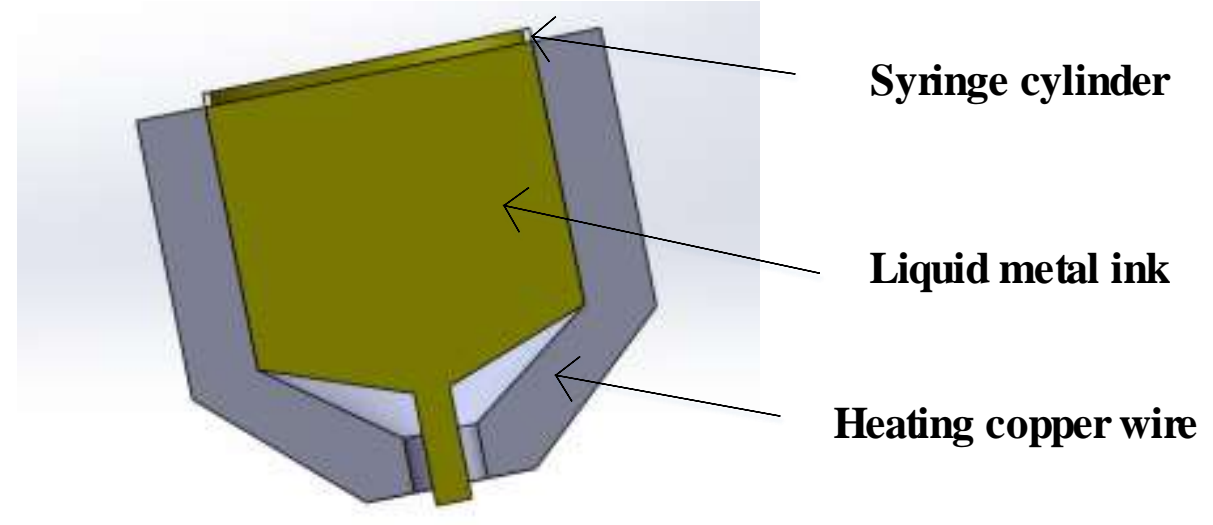

Figure 1. The structure of print head

For liquid 3D metal printing, all pure metals or alloys with melting points from about room temperature to about $300^{\circ} \mathrm{C}$ are likely to be used as 3D printed materials. In addition, a combination of metallic and non-metallic materials may be used to make a variety of printing materials. First introduced to the alloy as a 3D printing material, Bi35 In 48.6Sn15.9Zn0.4 alloy was selected as the printing material, the first of this functional material is prepared as follows: four kinds of metals (bismuth, indium, tin and zinc) with the purity above $99.99 \%$ were weighed according to the ratio of 35:48.6:16:0.4. These pure metals were put in a beaker for 5 hours at $245^{\circ} \mathrm{C}$ in an electric vacuum drying oven. Then, the mixture was stirred in the beaker which was put in water bath at $85-90^{\circ} \mathrm{C}$ for $30 \mathrm{~min}$. Finally, the beaker was kept in the electric vacuum drying oven at $245^{\circ} \mathrm{C}$ for 2 hours to further ensure a well-mixed alloy solution. This liquid metal easily clogs in the syringe needle during curing due to solidification. To solve this problem, the syringe is mounted in an aluminum alloy cylinder heated by a constant resistance wire, and the temperature controller is used to adjust the power supply to the resistance wire to maintain a constant temperature of the metal cylinder. Since the melting point of the alloy is slightly higher than room temperature and the degree of undercooling is low, the Bi35In48.6Sn15.9Zn0.4 in the liquid phase rapidly cools in the temperature range of $50-60^{\circ} \mathrm{C} 0$.

The liquid-phase 3D printing method using this material should consider several factors, first of all, the nature of the coolant: the temperature of the coolant will have a direct effect on the print result. If the temperature is set too high, the new droplets will melt with the previous ones, with the result that the resulting structure is hard to "grow". However, if the temperature is set too low, the drop will rapidly cool and solidify as the heat transfer process is completed immediately. Second, the air pressure and needle diameter inside the syringe barrel play an important role in this manufacturing process, both of which affect the size of the drop and the distance between two adjacent drops. Finally, the diameter of the droplet is also affected by the size of the needle, which increases as the needle size increases.

\section{Research Mixing Print Materials}

Hybrid Printing and Materials Introduction. Currently, mixed 3D printing is an important direction in the field of rapid prototyping, which combines various printing methods and printing materials to achieve better printing object performance such as making 3D polymer scaffolds. So 
far, owing to the large difference between the melting point of the conventional metallic inks and non-metallic materials, metallic and non-printing ink mixing together form the functional element is still a great challenge. A basic method of 3D structures and electronic devices composed of low-melting-point liquid metal and nonmetallic materials is proposed. As a hybrid printing concept, a three-layer tri-color LED stereophonic circuit is used to describe printing 00.

To ensure that the metal material is always in a liquid state prior to printing, the print head needs to be heated while the machine is running. For liquid metal pneumatically $3 \mathrm{D}$ printing method, introducing the above mentioned metal material Bi35In48.6Sn15.9Zn0.4, studies have shown that this material printed product having a metallic luster, description is not easily oxidized, and the above-mentioned preparation method of the material in detail.The melting temperature of the material and undercooling were of $58.3^{\circ} \mathrm{C}$ and $2.4^{\circ} \mathrm{C}$, respectively. This alloy can be used to complete solid-liquid phase transformation at a temperature in the range of $50-60^{\circ} \mathrm{C}$. The melting enthalpy and specific heat capacities of the Bi35In48.6Sn15.9Zn0.4 alloy were $28.94 \mathrm{~J} / \mathrm{g}$ and $0.262 \mathrm{~J} /\left(\mathrm{g}{ }^{\circ} \mathrm{C}\right)$, respectively, which were much smaller $\left(393.0 \mathrm{~J} / \mathrm{g}\right.$ and $0.88 \mathrm{~J} /\left(\mathrm{g}{ }^{\circ} \mathrm{C}\right)$, these characteristics allow the molten droplets of this alloy to be cooled in a very short time at room temperature 00 .

Method of Mixed Printing. Next, the mixed printing of metal and nonmetal materials with different physical properties will be introduced, and alloy Bi35In48.6Sn15.9Zn0.4 and 705 silicone rubber will be selected for metallic and non-metallic materials respectively. As we all know, 705 silicone rubber is a neutral transparent one-component RTV (room temperature vulcanized) silicone rubber, which at room temperature after a few air will be cured, non-toxic, non-corrosive, electrical insulation, arc discharge, can be used between $60-150^{\circ} \mathrm{C}$, with excellent adhesion properties. 705 Silicone rubber is self-adhering and does not require heating when applied as a material during printing,after curing for 3-30 minutes, its cure depth is $2-3 \mathrm{~mm}$ after 24 hours at room temperature and $50 \%$ relative humidity, and its good strength and flexibility prevent the seal components from being easily damaged 0 .At the beginning of the manufacturing process for the mixed objects, a few millimeters thick layer of silicone rubber was printed on the bottom of the plastic petri dish. The clear colloid became flat due to its self-leveling and completely cured under normal humidity after 24 hours. The metal structure is then printed on the surface of the first layer with a Bi35In48.6Sn15.9Zn0.4 alloy material and the printed structure is cured in a few seconds. After this step, a second layer of 705 silicone rubber was printed over the metal layer for a curing time similar to step 1, and finally, a print mixture having three layers of burger structure was removed from the plastic petri dish as shown in Fig.2. Printed products can be seen through the metal droplets and silicone rubber has better compatibility.

\begin{tabular}{c|c|c|c|c|c|c|c|c|}
\hline $\begin{array}{c}\text { Printing the } \\
\text { silicone rubber } \\
\text { layer, }\end{array}$ & Cooling & $\begin{array}{c}\text { Printing the } \\
\text { Pilicone rubber } \\
\text { layer }\end{array}$ & $\begin{array}{c}\text { Cool and solidify } \\
\text { layer }\end{array}$ & Take out
\end{tabular}

Figure 2. Step of printing

Phenomenon of Mixed Printing. 3D hybrid printing, in addition to these packaged metal structures and electronics, some of the packaged stereo circuits can be printed using 3D hybrid printing. For example, the three-layer LED stereo circuit is printed with Bi35In48.6Sn15.9Zn0.4 and 705 silicone rubber materials. The stereo circuit consists of six LEDs connected respectively to the current-limiting resistors 0. Before printing the metal layer, place the SMD component according to the position in the circuit layout with the reverse side up. During the printing process, it was found that the Bi35 In48.6Sn15.9Zn0.4 alloy could not be firmly connected to the SMD original copper electrode, in order to solve this problem the copper electrode needs to be pre-treated. First, a layer of tin (melting point of $183^{\circ} \mathrm{C}$ ) is attached to the electrode, thereby forming an intermetallic layer containing a $\mathrm{Cu}-\mathrm{Sn}$ alloy and then spreading a print material on the tin layer to form a mixed metal layer containing a metal element in both the print layer and the solder layer.The 
positive and negative electrodes of the printed circuit are switched on and the LEDs emit bright three colors, indicating that functional circuits with a spatial three-dimensional structure can be printed in a simple manner using low-melting-point metals and insulating packaging materials 0 .

\section{Conclusions}

In recent years, 3D printing technology has been developed rapidly, the study of 3D printing materials are also more in-depth, the current polymer materials due to lower melting point, becoming the most widely used materials, in the automotive, medical devices, consumer electronics, home appliances and other fields has been widely used, but for parts that require high strength and stiffness, polymer materials are powerless. The 3D metal printing, due to the higher melting point of the metal, and requires a higher degree of purity of the metal, so 3D metal printing costs are higher, the slow development of the field of metal printing. For 3D printing, rapid prototyping can only be better developed if the constraints on 3D printed materials are overcome. This article describes the liquid metal Bi35In48.6Sn15.9Zn0.4 alloy 3D metal printing applications, with low melting point, low cost, fast cooling and other characteristics, to solve the problem of the original high melting point metal materials, through the metal material Bi35In48.6Sn15.9Zn0 .4 and the non-metallic 705 silicone hybrid print stereo circuit, validating that this new alloy can be mixed with non-metallic materials for 3D printing and that the two show a high level of compatibility. By demonstrating the three-layer tri-color LED circuit to illustrate the potential of this hybrid printing method, it can be seen that future hybrid printing will be an important direction, the combination of low melting point metal and insulating packaging material provides a new direction for the development of 3D metal printing materials in the future.

\section{References}

[1] J.Y. Wang, L. Chai, L.B. Liu, X.R. Zhao, Y.F. Xu, X.W. Zhou, W.M. Zhang and X.H. Wang: Journal of Mechanical Engineering, Vol. 50 (2014) No.23, p 119.

[2] Y.S. Shi, L.C. Zhang, Y. Bai and Z.Y. Zhao: Scientia Sinica Informationis, Vol. 45 (2015) No.2, p. 197.

[3] X.Y. Song and J.F. Xing: CIESC Jorunal, Vol.66 (2015) No.9, p3324.

[4] D.J. Zhou. Electro-Mechanical Engineering, Vol. 33 (2017) No.2, p.13.

[5] C.Y. Wang and Q. Su:Journal of Intelligence, Vol. 34(2015) No.9, p71.

[6] Z.Q. Zhang, Z.L. Wang, Y.W. Chen, Z.X. Pang, L. Wang and C.C. Ma. Journal of Machine Design, Vol. 33 (2016) No.11, p.56.

[7] N. Zhang and F. Li: Journal of Machine Design, Vol. 30 (2013) No.7, p97.

[8] X.F. Liu and J.F. Huang: Chinese Journal of Construction Machinery, Vol.13 (2015) No.1, p.82.

[9] L. Wang and J. Liu: Science China Technological Sciences, Vol.57 (2014) No.11, p2089.

[10]X.L. Li, J.X. Ma, P. Li, Q. Chen and W.M. Zhou: Process Automation Instrumentation, Vol.35 (2014) No.1, p1.

[11]Z.D. Shan, L.N. Yang, F. Liu, W.J. Rong and Q. Liu: Journal of Central South University, Vol. 47 (2016) No.11, p3642.

[12] H.G. Liu, Q. Yang, G.F. Liu and Q. Liu: Journal of Intelligence, Vol.32 (2016) No.6, p40.

[13] Y.Q. Wang, J.X. Shen and H.Q. Wu: Journal of Aeronautical Materials, Vol.36 (2016) No.4, $\mathrm{p} 89$.

[14] R.J. Xu, Z.A. Li, L.Y. Zhu, K.L. Li and Q.J. Yang: Machine Design and Manufacturing Engineering, Vol.45 (2016) No.3, p11.

[15] W. Lei and L. Jing: Science China Technological Sciences, Vol.57 (2014) No.9, p1721. 